\title{
Long-term effect of galantamine on cognitive function in patients with Alzheimer's disease versus a simulated disease trajectory: an observational study in the clinical setting
}

\author{
This article was published in the following Dove Press journal: \\ Neuropsychiatric Disease and Treatment \\ 19 April 2017 \\ Number of times this article has been viewed
}

\section{Ryoko Nakagawa' \\ Takashi Ohnishi' \\ Hisanori Kobayashi' \\ Toshio Yamaoka ${ }^{2}$ \\ Tsutomu Yajima ${ }^{3}$ \\ Ai Tanimura ${ }^{4}$ \\ Toshiya Kato ${ }^{4}$ \\ Kazutake Yoshizawa'}

'Evidence Generation Department, Medical Affairs Division, ${ }^{2}$ Clinical

Data Management Department, R\&D

Division, ${ }^{3}$ Biostatistics Department,

Quantitative Science Division, ${ }^{4}$ Drug

Surveillance Department, R\&D

Division, Janssen Pharmaceutical K.K., Tokyo, Japan
Correspondence: Takashi Ohnishi Evidence Generation Department Medical Affairs Division, Janssen

Pharmaceutical K.K., 5-2,

Nishikanda 3-chome Chiyoda-ku,

Tokyo 101-0065, Japan

Tel $+81344 I I 5009$

$\mathrm{Fax}+8 I 344 I I 5086$

Email tohnish8@its.jnj.com
Background: Long-term maintenance of cognitive function is an important goal of treatment for Alzheimer's disease (AD), but evidence about the long-term efficacy of cholinesterase inhibitors is sparse. To evaluate the long-term efficacy and safety of galantamine for $\mathrm{AD}$ in routine clinical practice, we conducted a 72-week post-marketing surveillance study. The effect of galantamine on cognitive function was estimated in comparison with a simulated disease trajectory.

Patients and methods: Patients with mild-to-moderate AD received flexible dosing of galantamine (16-24 mg/day) during this study. Cognitive function was assessed by the mini mental state examination (MMSE) and the clinical status was determined by the Clinical Global Impression-Improvement (CGI-I). Changes of the MMSE score without treatment were estimated in each patient using Mendiondo's model. Generalized linear mixed model analysis was performed to compare the simulated MMSE scores with the actual scores.

Results: Of the 661 patients who were enrolled, 642 were evaluable for safety and 554 were assessed for efficacy. The discontinuation rate was $46.73 \%$. Cognitive decline indicated by the mean change of actual MMSE scores was significantly smaller than the simulated decline. Individual analysis demonstrated that $>70 \%$ of patients had better actual MMSE scores than their simulated scores. Significant improvement of CGI-I was also observed during the observation period. Adverse events occurred in $28.5 \%$ of patients and were serious in $8.41 \%$. The reported events generally corresponded with the safety profile of galantamine in previous studies.

Conclusion: These findings support the long-term efficacy of galantamine for maintaining cognitive function and the clinical state in $\mathrm{AD}$ patients. Treatment with galantamine was generally safe. Importantly, this study revealed that galantamine improved cognitive function above the predicted level in $>70 \%$ of the patients.

Keywords: Alzheimer's disease, cholinesterase inhibitor, cognitive function, disease progression, real-world evidence

\section{Introduction}

Alzheimer's disease (AD) is the most common type of dementia, affecting over 25 million people worldwide. ${ }^{1}$ At present, there are no treatments that can stop or reverse the progression of $\mathrm{AD}$, and current medications such as cholinesterase inhibitors (ChEIs) are only considered to be symptomatic therapy.

Several meta-analyses have consistently documented modest effects of ChEIs on cognitive function. ${ }^{2,3}$ However, these meta-analyses have mainly assessed placebocontrolled randomized trials that demonstrated relatively short-term effects on 
cognitive function, neuropsychiatric symptoms, and global clinical assessments. Because ethical constraints prohibit long-term placebo-controlled studies of ChEIs in AD, placebo-controlled trials of these drugs have generally not run for longer than 1 year. On the other hand, AD is a slowly progressive disease and patients can be expected to survive for an average of 8-10 years at the time of diagnosis, ${ }^{4}$ which means that long-term efficacy is clinically important. Based on our literature search, only two relatively long-term studies ( 2 years) have been conducted to assess the efficacy of ChEIs (donepezil and galantamine) in $\mathrm{AD}, 5,6$ which means there is little evidence regarding the long-term efficacy of these drugs. Due to this lack of robust evidence, the American Geriatric Society has stated that the risk and benefits of longterm ChEI therapy are not well established. ${ }^{7}$

Long-term open-label observational studies performed in the routine clinical setting can provide supplementary information to that demonstrated by placebo-controlled trials, particularly with regard to long-term efficacy. Because open-label observational studies lack a placebo arm, detailed understanding of the natural disease trajectory in patients with AD is important for analysis of the data. Several authors have proposed mathematical models for describing cognitive decline in untreated patients with $\mathrm{AD} \cdot{ }^{8-10} \mathrm{All}$ these models suggest that there is a significant quadratic correlation between baseline cognitive function and the annual rate of cognitive decline in untreated AD cohorts. ${ }^{8-10}$ Using such models, several studies have been conducted to assess the long-term efficacy of ChEIs for AD in comparison with the simulated untreated trajectory of cognitive decline and to establish models for prediction of expected changes in ChEI-treated patients. ${ }^{11,12}$ However, these studies involved prediction of group mean values rather than individual patient outcomes. Therefore, the long-term benefits of ChEI treatment for individual AD patients have not been clarified. Because of the modest effect of ChEIs on cognitive function in $\mathrm{AD}$ and considerable variability of the disease trajectory, it is difficult to judge whether ChEI therapy is beneficial on a patient-by-patient basis. To do so would require a reliable individual patient-based predictive model for cognitive outcomes in untreated AD. Such a model could support clinical decisions, such as switching to another ChEI because the current medication was predicted to lack efficacy.

We conducted a 72-week post-marketing surveillance study in the routine clinical setting to evaluate the efficacy and safety of galantamine therapy for AD based on the mini mental state examination (MMSE) and the Clinical Global
Impression Improvement scale (CGI-I). ${ }^{13}$ This study was designed to investigate the following points:

1. The long-term effect of galantamine on cognitive function (MMSE) and on the clinical state (CGI-I) in patients with mild-to-moderate AD during a 72-week treatment period.

2. The effect of galantamine on cognitive function in comparison with the untreated disease trajectory predicted by Mendiondo's mathematical model ${ }^{9}$ at both the group and individual patient level.

3. The safety and adverse effects of galantamine therapy in the routine clinical setting.

\section{Patients and methods Study design}

This was a 72-week multicenter observational open-label study of galantamine therapy for AD. Patients meeting the enrollment criteria were men or women with mild-tomoderate AD who had recently commenced treatment with galantamine. The diagnosis of AD was done based on each physician's clinical judgment and severity of AD was defined based on functional assessment staging of Alzheimer's disease and MMSE. Patients with a history of allergic reactions to ChEIs were excluded. Physicians were advised that all treatments and dose adjustments should be based on approved regimens, and management decisions were made at the attending physician's discretion according to routine practice. In general, treatment was started with $4 \mathrm{mg}$ b.i.d. and the dosage was increased to $8 \mathrm{mg}$ b.i.d. after 4 weeks. Then it was maintained at $8 \mathrm{mg}$ b.i.d. for at least 4 weeks before being increased to $12 \mathrm{mg}$ b.i.d. with dosage adjustment being based on the physician's assessment of the clinical response and tolerability. The study drug was not supplied to the patients and they received the medication as part of their usual care. The protocol of this study, including the ethical aspects, was assessed by an internal review board of Janssen Pharmaceutical (Named: Research Concept Review Board), and was approved by the Pharmaceuticals and Medical Devices Agency (PMDA). Conducting this post-marketing surveillance was a part of mandatory actions determined and reviewed by PMDA. The study was conducted in accordance with the Japanese regulation (Ministry of Health, Labour and Welfare Ministerial Ordinance No 171) of Good Post-marketing Study Practice. Each site (hospitals or clinic) follows its own regulations or standards for obtaining of the IRB approval, including taking either written or oral informed consent for participants at each site. 
A similar procedure is generally followed in Japan for this type of naturalistic registry study.

\section{Outcome assessment}

Cognitive function was assessed by using the $\mathrm{MMSE}^{14}$ and the global severity and/or change of the clinical condition was assessed by the CGI-I scale. The MMSE and CGI-I scores were determined at baseline and after 4, 12, 24, 36, 48, 60, and 72 weeks of treatment with galantamine. Adverse events (AEs) and data on patients who discontinued treatment were also recorded during the study period.

An electronic data capture system was used, allowing the majority of the data to be transcribed from source documents into the electronic case report forms by the attending physicians and transmitted securely to the sponsor.

\section{Models of the natural cognitive decline}

The natural course of cognitive decline without treatment was simulated by calculating changes of the MMSE score using a previously reported model. ${ }^{9}$

The original model and 95\% confidence limits reported by Mendiondo et al was:

Annual change of the MMSE score $=1 /\left(a x^{2}+b x+c\right)$, $a=0.00334 \pm 0.00048, b=0.0730 \pm 0.0136$,

$c=0.6013 \pm 0.0884 x=$ Baseline MMSE score

Because of the significant impact of age in this model, it was weighted according to the duration of cognitive decline for two age clusters as follows. ${ }^{9}$

$$
\begin{aligned}
& \text { Weight }_{<72 \text { years old }}=5.90^{2} /(5.90+8.23)=0.8351 \\
& \text { Weight }_{\geq 72 \text { years old }}=8.23^{2} /(5.90+8.23)=1.1649
\end{aligned}
$$

The confidence interval (CI) of the model was adjusted by Bonferroni's correction because of the significantly low probability that all of these three coefficients would take 95\% confidence limits. Thus, the final model used in this study was as follows.

$<72$ years old: annual change of the MMSE score $=$

$1 /\left(a x^{2}+b x+c\right), a_{<72 \text { years old }}=0.00334 \times 0.8351 \pm 0.00016$,

$b_{<72 \text { years old }}=0.00730 \times 0.8351 \pm 0.0045333$,

$c_{<72 \text { years old }}=0.6013 \times 0.8351 \pm 0.0294667$

$\geq 72$ years old: annual change of the MMSE score $=$

$1 /\left(a x^{2}+b x+c\right), a_{\geq 72 \text { years old }}=0.00334 \times 1.1649 \pm 0.00016$,

$b_{\geq 72 \text { years old }}=0.00730 \times 1.1649 \pm 0.0045333$,

$c_{\geq 72 \text { years old }}=0.6013 \times 1.1649 \pm 0.0294667$
Estimated 95\% CI for 6 months and 1 year determined by these equations with different baseline MMSE values are shown in Table 1. Based on this table, the number of patients was determined who had MMSE scores higher than the predicted upper limit of the CI (better than untreated),

Table I Estimated $95 \% \mathrm{Cl}$ of natural cognitive decline without

\begin{tabular}{|c|c|c|c|c|c|}
\hline \multirow{2}{*}{$\begin{array}{l}\text { Age, } \\
\text { years }\end{array}$} & \multirow{2}{*}{$\begin{array}{l}\text { Baseline } \\
\text { MMSE }\end{array}$} & \multicolumn{2}{|l|}{ Half year } & \multicolumn{2}{|l|}{ I year } \\
\hline & & Lower Cl & Upper Cl & Lower Cl & Upper Cl \\
\hline \multirow{22}{*}{$<72$} & 3 & 1.37 & 1.69 & -0.26 & 0.38 \\
\hline & 4 & 2.08 & 2.55 & 0.17 & 1.10 \\
\hline & 5 & 2.73 & 3.41 & 0.46 & 1.81 \\
\hline & 6 & 3.29 & 4.27 & 0.58 & 2.54 \\
\hline & 7 & 3.76 & 5.15 & 0.52 & 3.29 \\
\hline & 8 & 4.14 & 6.05 & 0.28 & 4.10 \\
\hline & 9 & 4.45 & 6.99 & -0.10 & 4.98 \\
\hline & 10 & 4.77 & 7.98 & -0.46 & 5.96 \\
\hline & 11 & 5.23 & 9.01 & -0.54 & 7.02 \\
\hline & 12 & 5.97 & 10.09 & -0.05 & 8.18 \\
\hline & 13 & 7.09 & 11.20 & 1.18 & 9.40 \\
\hline & 14 & 8.54 & 12.33 & 3.08 & 10.66 \\
\hline & 15 & 10.18 & 13.47 & 5.37 & 11.94 \\
\hline & 16 & 11.88 & $|4.6|$ & 7.76 & 13.22 \\
\hline & 17 & 13.53 & 15.75 & 10.07 & 14.50 \\
\hline & 18 & 15.10 & 16.88 & 12.20 & 15.75 \\
\hline & 19 & 16.57 & 17.99 & 14.15 & 16.98 \\
\hline & 20 & 17.96 & 19.09 & 15.92 & 18.19 \\
\hline & 21 & 19.27 & 20.19 & 17.54 & 19.37 \\
\hline & 22 & 20.52 & 21.27 & 19.04 & 20.54 \\
\hline & 23 & 21.73 & 22.34 & 20.46 & 21.68 \\
\hline & 24 & 22.90 & 23.40 & 21.79 & 22.81 \\
\hline \multirow{22}{*}{$\geq 72$} & 3 & 1.83 & 2.06 & 0.67 & 1.12 \\
\hline & 4 & 2.63 & 2.96 & 1.25 & 1.92 \\
\hline & 5 & 3.37 & 3.86 & 1.74 & 2.72 \\
\hline & 6 & 4.06 & 4.76 & 2.12 & 3.52 \\
\hline & 7 & 4.68 & 5.67 & 2.36 & 4.34 \\
\hline & 8 & 5.23 & 6.60 & 2.46 & 5.20 \\
\hline & 9 & 5.74 & 7.56 & 2.48 & 6.12 \\
\hline & 10 & 6.25 & 8.55 & 2.50 & 7.10 \\
\hline & 11 & 6.86 & 9.57 & 2.73 & 8.15 \\
\hline & 12 & 7.68 & 10.63 & 3.36 & 9.26 \\
\hline & 13 & 8.76 & |l.7| & 4.53 & 10.42 \\
\hline & 14 & 10.09 & 12.80 & 6.17 & 11.60 \\
\hline & 15 & 11.55 & 13.90 & 8.10 & $|2.8|$ \\
\hline & 16 & 13.05 & 15.00 & 10.10 & $|4.0|$ \\
\hline & 17 & 14.52 & 16.10 & 12.03 & $|5.2|$ \\
\hline & 18 & 15.92 & 17.19 & 13.84 & 16.39 \\
\hline & 19 & 17.26 & 18.28 & 15.52 & 17.55 \\
\hline & 20 & 18.54 & 19.35 & 17.07 & 18.70 \\
\hline & 21 & 19.76 & 20.42 & 18.52 & 19.83 \\
\hline & 22 & 20.94 & 21.48 & 19.88 & 20.95 \\
\hline & 23 & 22.09 & 22.53 & 21.18 & 22.05 \\
\hline & 24 & 23.21 & 23.57 & 22.42 & 23.14 \\
\hline
\end{tabular}
treatment according to the modified Mendiondo's model

Abbreviations: $\mathrm{Cl}$, confidence interval; MMSE, mini mental state examination. 
within the CI range, or below the predicted lower limit of the CI (worse than untreated) after 1 year and after 1.5 years of galantamine treatment.

\section{Statistical analysis}

The discontinuation rate was estimated by the Kaplan-Meier method, and comparison of the discontinuation rate between patients on monotherapy versus those on combined therapy was performed with the log-rank test. Comparison between simulated and actual changes of MMSE scores was performed with a generalized linear mixed model (GLMM). The actual baseline MMSE scores were used to calculate the predicted scores for each patient. To manage missing values, analysis was based on the observed cases (OC) at each scheduled visit and on data obtained by the last observation estimated forward (LOEF) method, which replaces missing values based on observed previous value and Mendiondo's model. Interactions of time with the type of data (predicted untreated outcome, actual OC, or actual LOEF) were modeled on GLMM. When significant differences were detected, post-hoc comparisons were performed with the Tukey-Kramer test.

A two-sided $P<0.05$ was considered to be statistically significant. Statistical analyses were conducted using
R Statistical Software, version 3.1.0 (Foundation for Statistical Computing, Vienna, Austria) and SAS software, version 9.3 (SAS Institute Inc., Cary, NC, USA). GLMM was performed with the GLIMMIX procedure of SAS and other analyses were performed using $\mathrm{R}$.

\section{Results}

\section{Baseline demographic and clinical characteristics}

A total of 661 patients were enrolled in this study, of whom 642 were evaluable for safety and 554 for efficacy (Figure 1). Seventy subjects were removed from the efficacy dataset because of lack of sufficient baseline information. The baseline demographic and clinical characteristics are summarized in Table 2 . In the safety analysis set, the mean \pm standard deviation (SD) age was 79.10 \pm 7.20 years, the mean disease duration was 0.73 years, and $4.98 \%$ of the patients were hospitalized at study entry. Among the subjects, $22.90 \%$ were using other anti-dementia drugs before study entry. According to the functional assessment of staging scale, most patients had mild (39.10\%) or moderate $(47.66 \%) \mathrm{AD}$ at study entry, while the mean MMSE score was $18.95 \pm 5.04$. The mean daily dose of galantamine was $15.01 \pm 4.76 \mathrm{mg}$ and

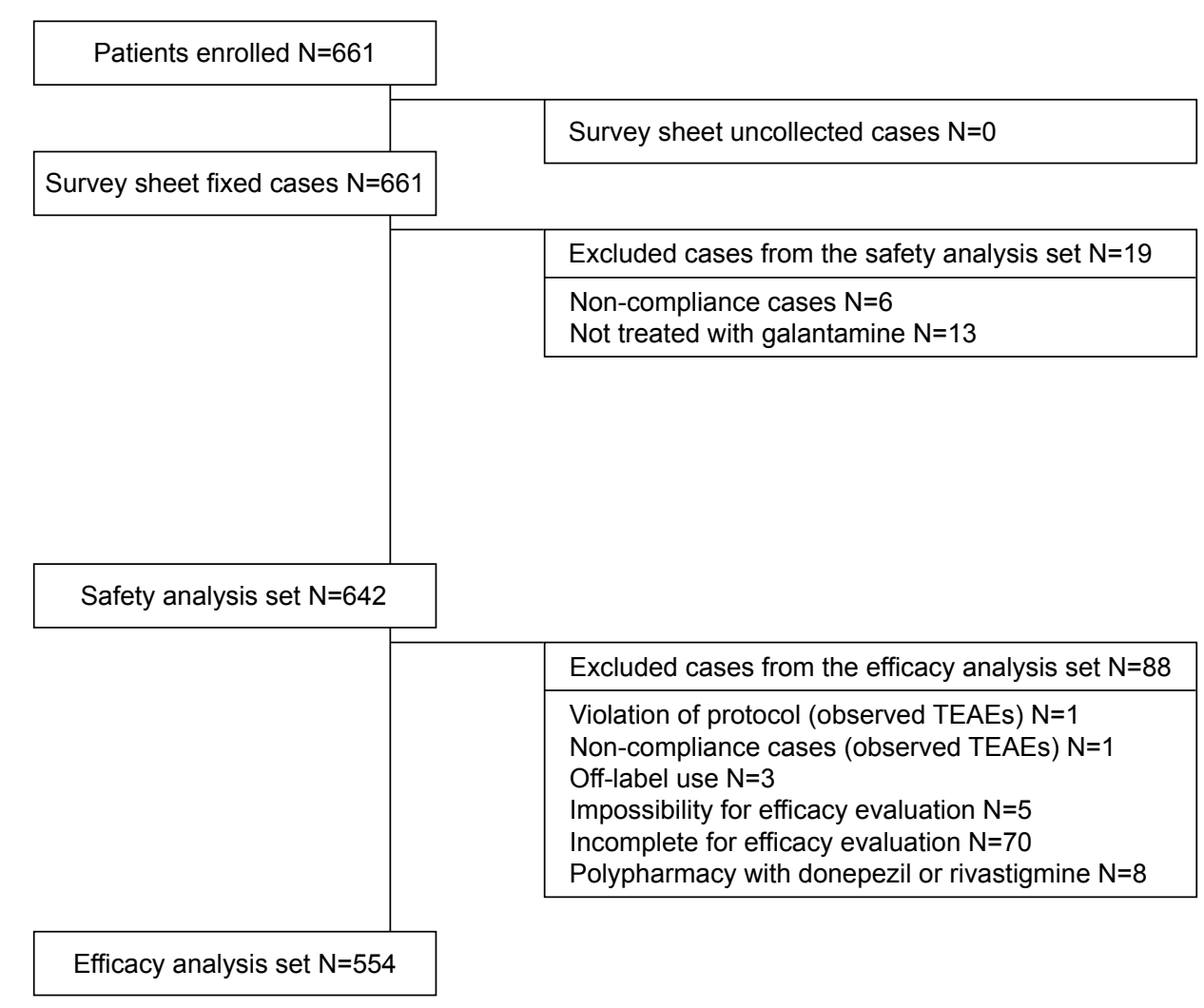

Figure I Flow chart of subject disposition.

Abbreviation: TEAEs, treatment emergent adverse events. 
Table 2 Baseline demographic and clinical characteristics

\begin{tabular}{|c|c|c|}
\hline & $\begin{array}{l}\text { Patients included in } \\
\text { the safety analysis }\end{array}$ & $\begin{array}{l}\text { Patients included in } \\
\text { the efficacy analysis }\end{array}$ \\
\hline Characteristic & $\mathrm{N}=642$ & $\mathrm{~N}=554$ \\
\hline Age (years), mean (SD) & $79.10(7.20)$ & $78.99(7.31)$ \\
\hline$<65, \mathrm{n}(\%)$ & $21(3.27 \%)$ & $19(3.43 \%)$ \\
\hline $65-74, \mathrm{n}(\%)$ & $126(19.63 \%)$ & $113(20.40 \%)$ \\
\hline$\geq 75, \mathrm{n}(\%)$ & 495 (77.10\%) & $422(76.17 \%)$ \\
\hline \multicolumn{3}{|l|}{ Gender, n (\%) } \\
\hline Female & $421(65.58 \%)$ & $369(66.61 \%)$ \\
\hline Male & $221(34.42 \%)$ & 185 (33.39\%) \\
\hline Disease duration (year), mean (SD) & $0.73(1.48)$ & $0.75(I .4 I)$ \\
\hline \multicolumn{3}{|l|}{ Hospitalization at study entry, $\mathrm{n}(\%)$} \\
\hline No & $566(88.16 \%)$ & $486(87.73 \%)$ \\
\hline Yes & $32(4.98 \%)$ & $26(4.69 \%)$ \\
\hline Repeated entry and leave & $44(6.85 \%)$ & $42(7.58 \%)$ \\
\hline Dose (mg/day), mean (SD) & $|5.0|(4.76)$ & $15.52(4.48)$ \\
\hline Duration of exposure (days), mean (SD) & $343.44(202.30)$ & $371.74(185.63)$ \\
\hline \multicolumn{3}{|l|}{ Pretreatment antidementia drugs, $\mathrm{n}(\%)$} \\
\hline No & $495(77.10 \%)$ & $425(76.72 \%)$ \\
\hline Yes & 147 (22.90\%) & $129(23.29 \%)$ \\
\hline Polypharmacy, n (\%) (combined with memantine) & $65(10.12 \%)$ & $60(10.83 \%)$ \\
\hline \multicolumn{3}{|l|}{ Stages of Alzheimer's disease (FAST stage) } \\
\hline No cognitive decline, $n(\%)$ & $0(0.00 \%)$ & $0(0.00 \%)$ \\
\hline Very mild cognitive decline, $\mathrm{n}(\%)$ & $21(3.27 \%)$ & $20(3.61 \%)$ \\
\hline Mild cognitive decline, $n(\%)$ & $251(39.10 \%)$ & $209(37.73 \%)$ \\
\hline Moderate cognitive decline, $n(\%)$ & $306(47.66 \%)$ & $271(48.92 \%)$ \\
\hline Moderately severe cognitive decline, $\mathrm{n}(\%)$ & $53(8.26 \%)$ & $46(8.30 \%)$ \\
\hline Severe cognitive decline, $n(\%)$ & $2(0.31 \%)$ & $2(0.36 \%)$ \\
\hline Very severe cognitive decline, $\mathrm{n}(\%)$ & I $(0.16 \%)$ & $\mathrm{I}(0.18 \%)$ \\
\hline MMSE, mean (SD) & - & $18.95(5.04)$ \\
\hline
\end{tabular}

Abbreviations: FAST, functional assessment staging; MMSE, mini mental state examination; SD, standard deviation.

the mean duration of drug exposure was $343.44 \pm 202.30$ days. Sixty-five patients $(10.12 \%)$ received memantine combined with galantamine (memantine users).

\section{Discontinuation rate}

Discontinuation during the observation period is summarized in Figure 2. The discontinuation rate was $\sim 20 \%$ at 12 weeks, $40 \%$ at 48 weeks, and $46.73 \%$ after 72 weeks (Figure 2). Patients discontinued the study because of safety problems (31.08\%), transfer to another hospital (24.32\%), loss of contact $(19.93 \%)$, personal choice $(11.15 \%)$, and other reasons (13.51\%). Post-hoc comparison between memantine users and non-users demonstrated a significantly lower discontinuation rate among memantine users than non-users (log-rank test, $P=0.0051$; Figure 2).

\section{Long-term effect of galantamine on cognitive function}

Changes of the MMSE score during the observation period are shown in Figure 3. The mean change from baseline to 72 weeks was 0.71 according to OC analysis, 1.21 according to LOEF analysis, and 3.35 for the predicted outcome without treatment. GLMM revealed a significant interaction between these three cohorts and time $(\mathrm{F}(2,6427)=14.63$, $P<0.001)$. A post-hoc Tukey-Kramer test identified significant differences between the $\mathrm{OC}$ and predicted outcomes and between the LOEF and predicted outcomes at 36 weeks (OC vs predicted: $t(3,403)=4.37, P=0.003$; LOEF

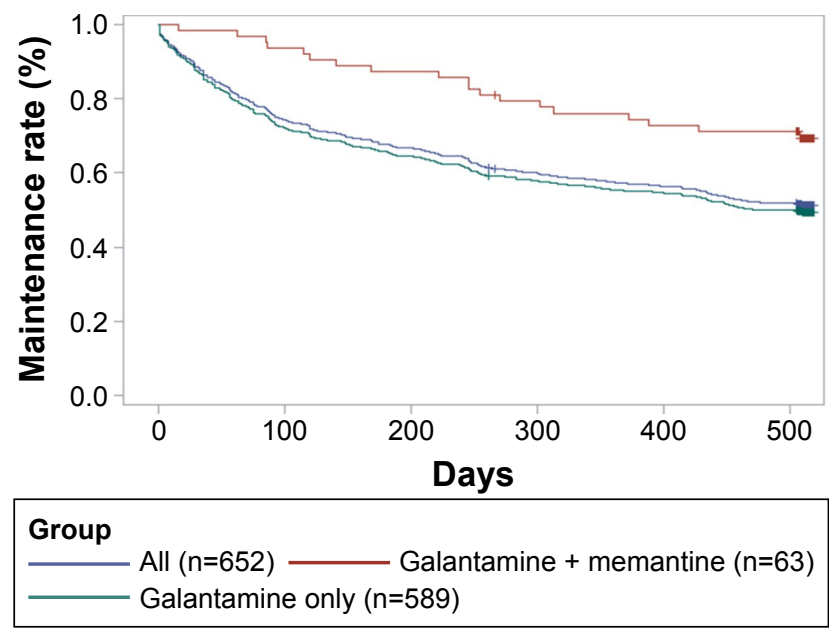

Figure 2 Kaplan-Meier curves for discontinuation during the observation period. 


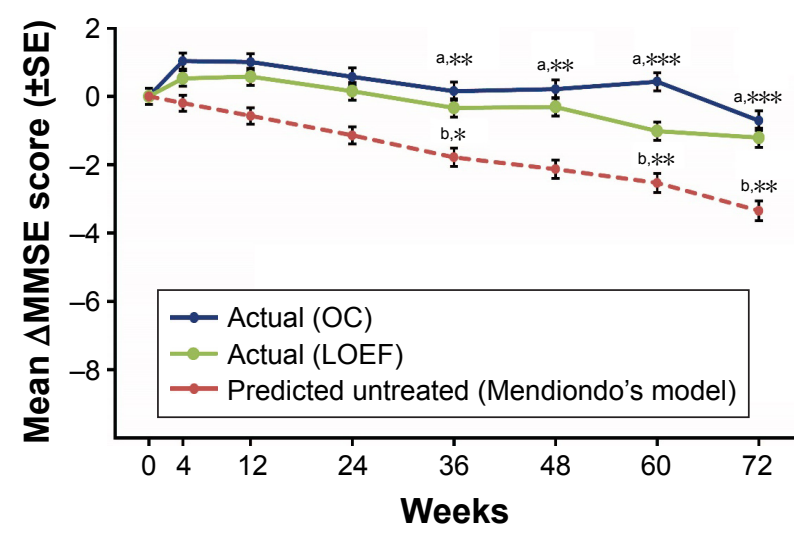

Figure 3 Mean actual changes of MMSE scores and simulated changes during the observation period.

Notes: Bars represent the standard error. GLMM analysis with post-hoc testing: a) differences between actual $O C$ and predicted scores, b) differences between actual, LOEF, and predicted scores. Asterisks denote significant differences: $* P<0.05$, $* * P<0.0$ I, *** $P<0.00$ I.

Abbreviations: GLMM, generalized linear mixed model; LOEF, last observation estimated forward; MMSE, mini mental state examination; OC, observed case.

vs predicted: $t(3,403)=3.96, P=0.016)$, at 48 weeks (OC vs predicted: $t(3,403)=4.49, P=0.0018$; LOEF vs predicted: $t(3,403)=3.59, P=0.0599)$, at 60 weeks (OC vs predicted: $t(3,403)=5.53, P<0.001$; LOEF vs predicted: $t(3,403)=4.16$, $P=0.0074)$, and at 72 weeks (OC vs predicted: $t(3,403)=5.45$, $P<0.0001$; LOEF vs predicted: $t(3,403)=4.54, P=0.0014)$. Table 3 displays the patients whose MMSE scores were above, within, or below the predicted CI. After 1 year, OC analysis showed that $75.65 \%$ of patients had an MMSE score significantly above the predicted value without treatment, as did $71.43 \%$ of patients at 1.5 years. According to LOEF analysis, $57.79 \%$ of patients had an MMSE score significantly above the predicted value after 1 year and $55.75 \%$ at 1.5 years. The time course of the observation period in CGI-I is shown in Figure 4. At 1.5 years, $2.24 \%$ of patients were rated as "very much improved", 10.45\% were "much improved", 32.46\% were "minimally improved", 33.21\% were "no change", 14.93\% were "minimally worse", 5.22\% were "much worse", and $1.12 \%$ were "very much worse".

\section{Safety}

At least one AE occurred in $28.50 \%$ of the patients. Table 4 shows AEs that occurred in $>1 \%$ of the patients. The most frequently reported AEs were nausea (5.30\%), decreased appetite $(3.43 \%)$, vomiting $(2.49 \%)$, insomnia $(1.40 \%)$, agitation (1.09\%), dizziness (1.09\%), and headache (1.09\%). At least one serious AE (SAE) occurred in $8.41 \%$ of the patients. Frequently reported SAEs occurring in at least two patients and the number of deaths are also listed in Table 4. The most common SAE was delusions (0.62\%). Twelve deaths $(1.87 \%)$ were reported during the study, with the causes being bronchial pneumonia $(n=1,0.16 \%)$, acute bronchitis $(n=1,0.16 \%)$, stomach cancer $(n=1,0.16 \%)$, aspiration pneumonitis $(n=1,0.16 \%)$, myocardial infarction/pneumonia $(n=1,0.16 \%)$, complete heart block $(n=1,0.16 \%)$, renal insufficiency $(n=1,0.16 \%)$, sepsis $(n=1,0.16 \%)$, drowning $(n=1$, $0.16 \%)$, and unknown $(\mathrm{n}=3,0.47 \%)$.

\section{Discussion}

The present study was designed to assess the long-term efficacy of galantamine in patients with mild-to-moderate AD in comparison with the natural disease trajectory predicted using a mathematical model, as well as investigating the safety and tolerability of galantamine therapy for AD in the real-world clinical setting.

We found that the mean MMSE score improved over 24 weeks and was stable up to 1 year. Furthermore, there was a long-term beneficial effect on cognitive function measured by the MMSE compared with the predicted outcome without treatment. When efficacy was assessed on a patient-by-patient basis, about $71 \%$ of the patients showed significantly better cognitive function at 72 weeks compared with the predicted outcome. In agreement with the changes of the MMSE score, assessment of the CGI-I score also showed that galantamine treatment was effective for maintaining the clinical state (better or unchanged) in almost $80 \%$ of the patients at 72 weeks. Taken together, these findings suggest

Table 3 Distribution of patient outcomes

\begin{tabular}{lllll}
\hline & Total, $\mathbf{n}$ & $\begin{array}{l}\text { Significantly } \\
\text { improved than the } \\
\text { prediction, } \mathbf{n}(\%)\end{array}$ & $\begin{array}{l}\text { Within natural } \\
\text { disease trajectory, } \\
\mathbf{n}(\%)\end{array}$ & $\begin{array}{l}\text { Significantly } \\
\text { worse than the } \\
\text { prediction, } \mathbf{n}(\%)\end{array}$ \\
\hline $\begin{array}{l}\text { OC } \\
\text { I year }\end{array}$ & 115 & & & $15(13.04)$ \\
$\begin{array}{l}\text { I.5 years } \\
\text { LOEF }\end{array}$ & 147 & $87(75.65)$ & $27(18.37)$ & $13(11.30)$ \\
I year & 353 & $105(71.43)$ & $15(10.20)$ & $41(11.61)$ \\
I.5 years & 348 & $204(57.79)$ & $108(30.59)$ & $36(10.34)$ \\
\hline
\end{tabular}

Abbreviations: OC, observed cases; LOEF, last observation estimated forward. 


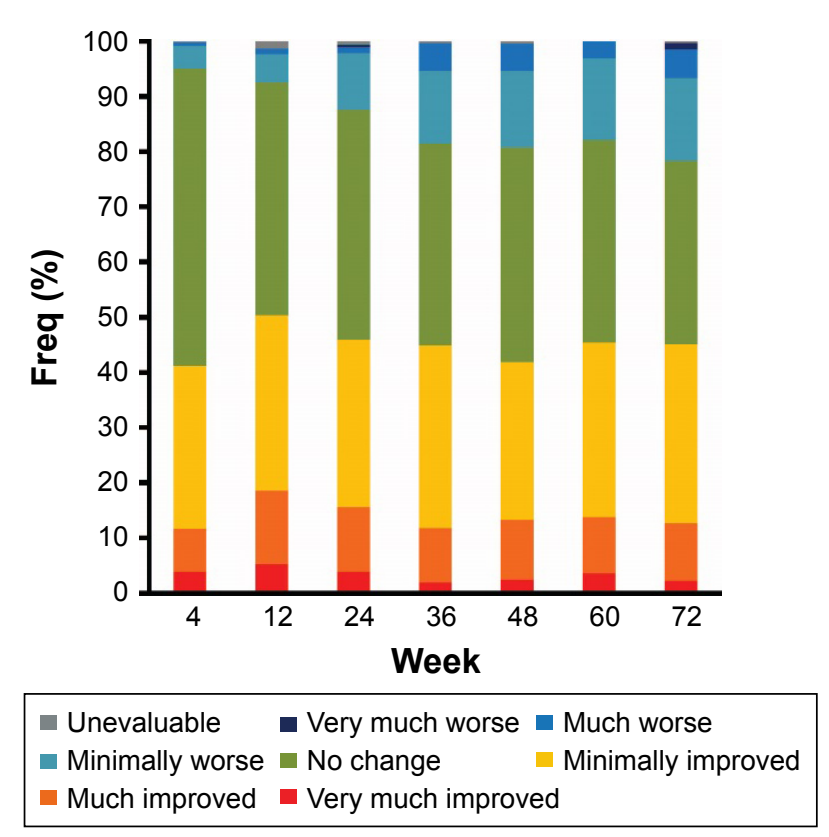

Figure 4 Clinical Global Impression-Improvement score over time during the observation period.

that galantamine therapy could be beneficial for $70 \%-80 \%$ of $\mathrm{AD}$ patients. Since about $10 \%$ of the patients received memantine in addition to galantamine, we also analyzed the data after excluding memantine users, but we found that the changes of the MMSE score were essentially the same

Table 4 Adverse events (AEs) and serious AEs

\begin{tabular}{ll}
\hline Adverse drug reaction & All patients, $\mathbf{N}=\mathbf{6 4 2}$ \\
& $\mathbf{N}(\%)$ \\
\hline Total number of patients with $\geq$ I\% AEs & $183(28.5)$ \\
Decreased appetite & $22(3.43)$ \\
Agitation & $7(1.09)$ \\
Insomnia & $9(1.40)$ \\
Dizziness & $7(1.09)$ \\
Headache & $7(1.09)$ \\
Diarrhea & $7(1.09)$ \\
Nausea & $34(5.30)$ \\
Vomiting & $16(2.49)$ \\
Total number of patients with SAEs & $54(8.41)$ \\
SAEs occurring in $\geq 2$ of all patients & \\
Delusion & $4(0.62)$ \\
Pneumonia & $2(0.31)$ \\
Hallucination & $2(0.31)$ \\
Altered state of consciousness & $2(0.31)$ \\
Dementia Alzheimer's type & $2(0.31)$ \\
Epilepsy & $2(0.31)$ \\
Pneumonia aspiration & $2(0.31)$ \\
Blood pressure decreased & $2(0.31)$ \\
Blood pressure increased & $2(0.31)$ \\
Spinal compression fracture & $2(0.31)$ \\
Total number of deaths & $12(1.87)$ \\
\hline
\end{tabular}

Note: All AEs occurring in $\geq 1 \%$ of patients and SAEs occurring more than once are shown.

Abbreviations: $A E$, adverse events; $S A E$, serious adverse events. (data not shown). On the other hand, the dropout rate was significantly higher among non-users than among memantine users. The indication for memantine therapy is moderateto-severe AD in Japan and the mean baseline MMSE score of memantine users was significantly lower than that of non-users (mean \pm SD: $16.79 \pm 5.41$ for memantine users vs $19.13 \pm 4.97$ for memantine non-users, $t$ - $(427)=2.43$, $P=0.02)$. The lower dropout rate of memantine users in the present study is inconsistent with the results of a previous study that suggested baseline disease severity and age were associated with dropout from AD trials. ${ }^{15}$ However, we have no adequate explanations for such a discrepancy at present. Safety evaluation revealed that all of the reported AEs were known events, such as gastrointestinal symptoms. Serious cardiovascular AEs were observed in $0.78 \%$ of the patients. There were no unpredictable or rare AEs, including Pisa syndrome. Accordingly, both efficacy and safety data supported the validity of long-term galantamine therapy for mild-to-moderate AD.

This study not only demonstrated a long-term beneficial effect of galantamine therapy for $\mathrm{AD}$ at the group level, but also revealed that galantamine maintained cognitive function of individual patients in the routine clinical setting. Published consensus recommendations and clinical practice guidelines advocate an individualized approach to $\mathrm{AD}$, but there is a need for more evidence that long-term therapy is worthwhile. ${ }^{16,17}$ As shown in Table 1, our present findings could be useful for assisting physicians and the relatives of patients when deciding treatment options. A previous study established a patient-level AD model and simulation method by applying beta regression to the Alzheimer's disease assessment scale cognitive subscale (ADAS-cog). ${ }^{18}$ While this beta regression model employing ADAS-cog could be suitable for clinical trials of disease-modifying drugs, it is complicated and ADAS-cog is not usually employed in the routine clinical setting. On the other hand, simulating changes of the MMSE score by using Mendiondo's model is simple and can be easily applied to individual patients in routine practice.

Similar to the present results, two previous placebocontrolled studies have demonstrated long-term beneficial effects of ChEI therapy. ${ }^{6,19}$ On the other hand, the Alzheimer's Disease Neuroimaging Initiative (ADNI) and the Australian Imaging, Biomarkers and Lifestyle study obtained opposing results, with greater cognitive decline amongst $\mathrm{AD}$ patients taking ChEIs compared with those not using ChEIs. ${ }^{20}$ Unlike the aforementioned two randomized controlled trials (RCTs) showing a favorable effect of ChEIs, the ADNI study was not a randomized trial, so treatment selection bias should be taken 
into consideration when interpreting its results (ie, physicians might have selectively prescribed ChEIs for patients with more progressive AD). We consider that long-term ChEI therapy is generally beneficial for AD patients.

As mentioned above, the present study demonstrated similar efficacy to that found in a previous 2-year placebocontrolled RCT of galantamine, ${ }^{6}$ but there were some discrepancies between our results and previous findings. Although similar patient factors were considered to be associated with cognitive decline (baseline MMSE and age), the previous study demonstrated a more rapid decrease of MMSE scores in patients receiving galantamine treatment compared with our results. It is possible that survival bias contributed to the favorable effect of galantamine in the present study. Observational studies have an inherent risk of survival bias due to dropout and high dropout rates are a problem in all long-term studies of AD. In this study, the completion rate was actually higher than in the previous study; however, it should be taken into consideration as different criteria for the termination: previous study has been terminated due to a Data Safety Monitoring Board-recommended early study termination. According to Wallin et al, the reported 1-year completion rate ranges widely from $26 \%$ to $89 \%$. $^{12}$ The completion rate was $53 \%$ at 1.5 years in the present study, which is average for this type of study. When changes of the MMSE score were assessed by OC analysis, the MMSE score was evaluated at 72 weeks in only $38.2 \%$ of the patients; so, there is a potential risk of survival bias. Since the last observation carried forward method seems to be inappropriate for analysis of MMSE decline in patients with a progressive disease like AD, we predicted MMSE decline based on Mendiondo's model and applied the results to dropouts as a supplementary analysis. The results of our group level LOEF analysis were essentially the same as those of OC analysis. On the other hand, there were an increased number of patients within the disease trajectory due to the relatively high discontinuation rate. However, $>50 \%$ of patients still showed significantly better cognitive function compared with the predicted outcome. While we cannot completely exclude survival bias, we believe that our data indicate a long-term beneficial effect of galantamine treatment on cognitive function. Indeed, a previous long-term randomized head-to-head comparison of galantamine with donepezil and an observational study with a relatively high completion rate demonstrated similar MMSE changes to those in our study, with stabilization of MMSE scores after 1 year of treatment. ${ }^{12,21}$ Another important difference between the present findings and those of the previous 2 -year study of galantamine therapy ${ }^{6}$ is that the decline of MMSE scores predicted by Mendiondo's model was more rapid than that observed in the placebo group of the previous study. Because the factors associated with Mendiondo's model (age and baseline MMSE score) were similar in both studies, this discrepancy should need to be explained. One possible explanation is the placebo effect in patients allocated to the placebo arm in the previous study. Since Mendiondo investigated the natural history of $\mathrm{AD}$ in an observational study without pharmacological intervention for dementia, the placebo effect on MMSE changes should have been negligible in their data. On the other hand, MMSE changes of participants in the previous placebo-controlled RCT would probably be modulated by the placebo effect and this might have contributed to the discrepancy with our present findings. Another possibility is that Mendiondo's mathematical model overestimates cognitive decline in untreated patients due to general advances in medicine since the 1980s, when the data set for the mathematical model was acquired. For example, an association between vascular risk factors, such as hypertension, diabetes, and cardiovascular disease, and the pathogenesis and/or progression of $\mathrm{AD}$ received attention only since around $2000 .^{22}$ In elderly people, vascular risk factors are currently managed better than in the 1980 s. This could affect the disease trajectory of AD and lead to overestimation of cognitive decline in untreated patients due to changes in the healthcare environment. Indeed, a previous pooled analysis of studies on donepezil conducted between 1990 and 1999 revealed that patients allocated to placebo therapy showed slower cognitive decline in more recent trials compared with older trials, despite having more comorbidities. ${ }^{23}$ Furthermore, our previous network metaanalysis of ChEI therapy revealed an increasing placebo effect on cognitive function over time across various drugs of this class. ${ }^{24}$ These results suggest that there may have been possible overestimation of the speed of cognitive decline based on Mendiondo's model in the present study. However, other studies conducted in the 2000s have revealed similar or more rapid decline of MMSE scores than the present data. ${ }^{5,25}$ For example, a study of the natural history of AD over 3 years reported by Holmes and Lovestone demonstrated annual decline of the MMSE score by 3.4 points (SD 3.5: median $=3.0$ ) in patients with mild-to-moderate disease. ${ }^{25}$ This reported rate of decline is larger than the decline simulated by Mendiondo's model in this study, but Holmes and Lovestone investigated a relatively small sample. ${ }^{25}$ Further research is needed to establish a contemporary disease trajectory model of AD for evaluating the efficacy of ChEI therapy and better management of AD patients.

Some limitations of the present study should be mentioned. First, the diagnosis of $\mathrm{AD}$ was done based on 
physician's clinical judgment rather than the strict diagnostic criteria. One would be concerned about the diagnostic accuracy of this study; however, the purpose of the study is to show effectiveness of galantamine in the real world. In this context, diagnosis based on physician's clinical judgment seems to be suitable for the purpose of this study. Second, several important patient characteristics associated with $\mathrm{AD}$ progression and risk were not investigated, including apolipoprotein E (ApoE) alleles and education level. While the influence of the $\varepsilon 4$ allele on the risk of $\mathrm{AD}$ and its age of onset is generally consistent in most studies, there have been widely varying reports with regard to whether different ApoE alleles influence the rate of cognitive decline following the onset of dementia. ${ }^{26}$ Mendiondo's model does not require information on ApoE alleles and we do not consider the lack of allele data to be a critical flaw. A lower education level is also considered to be a risk factor for developing $\mathrm{AD}$ and rapid progression. ${ }^{9,27}$ However, Wattmo et al reported an opposite effect of education level on the long-term outcome of $\mathrm{AD}$, with a high education level being a risk factor for faster cognitive decline. ${ }^{28}$ Thus, the relationship between education and disease progression remains to be clarified. Furthermore, the influence of education level on Mendiondo's model is smaller than that of age, so lack of education level data in this study should not be a critical flaw.

\section{Conclusion}

This 1.5-year observational study revealed long-term efficacy of galantamine for maintaining cognitive function in AD patients based on changes of the MMSE score, and also showed that galantamine therapy was generally safe and well tolerated. Importantly, individual analysis demonstrated that galantamine was beneficial for $>70 \%$ of $\mathrm{AD}$ patients.

\section{Acknowledgments}

This study was registered at umin.ac.jp (UMIN000017151). This study was funded by Janssen Pharmaceutical K.K., a division of Johnson \& Johnson, Japan.

\section{Author contributions}

All authors contributed to interpretation of the results, developed the draft manuscript, participated in subsequent revisions, and read and approved the final manuscript.

\section{Disclosure}

All authors are full-time employees of Janssen Pharmaceutical K.K., a division of Johnson \& Johnson, Japan. The authors report no other conflicts of interest in this work.

\section{References}

1. Brookmeyer R, Johnson E, Ziegler-Graham K, Arrighi HM. Forecasting the global burden of Alzheimer's disease. Alzheimers Dement. 2007;3: 186-191.

2. Birks J. Cholinesterase inhibitors for Alzheimer's disease. Cochrane Database Syst Rev. 2006;(1):CD005593.

3. Hansen RA, Gartlehner G, Webb AP, et al. Efficacy and safety of donepezil, galantamine, and rivastigmine for the treatment of Alzheimer's disease: a systematic review and meta-analysis. ClinInterv Aging. 2008; 3(2):211-225.

4. Wolfson C, Wolfson DB, Asgharian M, et al. A reevaluation of the duration of survival after the onset of dementia. N Engl J Med. 2001; 344(15):1111-1116.

5. Courtney C, Farrell D, Gray R, et al. AD2000 Collaborative Group. Longterm donepezil treatment in 565 patients with Alzheimer's disease(AD2000): randomised double-blind trial. Lancet. 2004;363(9427):2105-2115.

6. Hager K, Baseman AS, Nye JS, et al. Effects of galantamine in a 2-year, randomized, placebo-controlled studyin Alzheimer's disease. Neuropsychiatr Dis Treat. 2014;10:391-401.

7. American Geriatrics Society. Choosing wisely - five things physicians and patients should question: part 2 [Internet]. New York (NY): American Geriatrics Society; 2014 [cited Jun 23, 2014]. Available from: http://www.americangeriatrics.org/files/documents/5things_list_ PART.pdf. Accessed August 15, 2015.

8. Stern RG, Mohs RC, Davidson M, et al. A longitudinal study of Alzheimer's disease: measurement, rate, and predictors of cognitive deterioration. Am J Psychiatry. 1994;151(3):390-396.

9. Mendiondo MS, Ashford JW, Kryscio RJ, Schmitt FA. Modelling Mini-MentalState Examination changes in Alzheimer's disease. Stat Med. 2000;19:1607-1616.

10. Ashford JW, Kolm P, Colliver JA, et al. Alzheimer patient evaluation and the mini-mental state: Item characteristic curve analysis. J Gerontol. 1989;44(5):139-146.

11. Wattmo C, Hansson O, Wallin AK, Londos E, Minthon L. Predicting long-term cognitive outcome with new regression models in donepezil-treated Alzheimer patients in a naturalistic setting. Dement Geriatr Cogn Disord. 2008;26(3):203-211.

12. Wallin $\AA \mathrm{K}$, Wattmo C, Minthon L. Galantamine treatment in Alzheimer's disease: response and long-term outcome in a routine clinical setting. Neuropsychiatr Dis Treat. 2011;7:565-576.

13. Janssen Pharmaceutical K.K., Japan. Special Drug Use-results Survey for Long-term Administration of Invega $3 \mathrm{mg} / 6 \mathrm{mg} / 9 \mathrm{mg}$ Tablet. Available from: https://upload.umin.ac.jp/cgi-open-bin/ctr/ctr_view. cgi?recptno=R000019898. Accessed October 19, 2016.

14. Folstein MF, Folstein SE, McHugh PR. Mini-Mental State: a practical method for grading the cognitive state of patients for the clinician. J Psychiatr Res. 1975;12(3):189.

15. William-Faltaos D, Chen Y, Wang Y, Gobburu J, Zhu H. Quantification of disease progression and dropout for Alzheimer's disease. Int J ClinPharmacolTher. 2013;51(2):120-131.

16. National Institute for Health and Clinical Excellence (NICE). Donepezil, galantamine, rivastigmine and memantine for the treatment of Alzheimer's disease. NICE technology appraisal guidance 217. London, UK (GB): NICE; 2011. Available from: https://www.nice.org.uk/guidance/ta217. Accessed August 15, 2015.

17. Ihl R, Frolich L, Winblad B, Schneider L, Burns A, Moller HJ. World Federation of Societies of Biological Psychiatry (WFSBP) guidelines for the biological treatment of Alzheimer's disease and other dementias. World J Biol Psychiatry. 2011;12(1):2-32.

18. Rogers JA, Polhamus D, Gillespie WR, et al. Combining patient-level and summary-level data for Alzheimer's disease modeling and simulation: a beta regression meta-analysis. J Pharmacokinet Pharmacodyn. 2012;39:479-498.

19. Courtney C, Farrell D, Gray R, et al. Long-term donepezil treatment in 565 patients with Alzheimer's disease (AD2000): randomized doubleblind trial. Lancet. 2004;26;363(9427):2105-2115. 
20. Schneider LS, Insel PS, Weiner MW. Treatment with cholinesterase inhibitors and memantine of patients in the Alzheimer's disease neuroimaging initiative. Arch Neurol. 2011;68(1):58-66.

21. Wilcock G, Howe I, Coles H, et al. GALGBR-2 Study Group. A longterm comparison of galantamine and donepezil in the treatment of Alzheimer's disease. Drugs Aging. 2003;20:777-789.

22. Breteler MM. Vascular risk factors for Alzheimer's disease: an epidemiologic perspective. Neurobiol Aging. 2000;21(2):153-160.

23. Jones RW, Schwam E, Wilkinson D, et al. Rates of cognitive change in Alzheimer's disease: observations across a decade of placebo-controlled clinical trials with donepezil. Alzheimer Dis Assoc Disord. 2009;23(4): 357-364.

24. Kobayashi H, Ohnishi T, Nakagawa R, Yoshizawa K. The comparative efficacy and safety of cholinesterase inhibitors in patients with mildto-moderate Alzheimer's disease: a Bayesian network meta-analysis. Int J Geriatr Psychiatry. 2016;31(8):892-904.
25. Holmes $\mathrm{C}$, Lovestone $\mathrm{S}$. Long-term cognitive and functional decline in late onset Alzheimer's disease: therapeutic implications. Age Ageing. 2003;32(2):200-204.

26. Kim J, Basak JM, Holtzman DM. The role of apolipoprotein E in Alzheimer's disease. Neuron. 2009;63:287-303.

27. Segal-Gidan F, Cherry D, Jones R, Williams B, Hewett L, Chodosh J. Alzheimer's disease management guideline: update 2008. Alzheimers Dement. 2011;7(3):e51-e59.

28. Wattmo C, Wallin AK, Londos E, Minthon L. Predictors of long-term cognitive outcome in Alzheimer's disease. Alzheimers Res Ther. 2011; 3(4):23.

\section{Publish your work in this journal}

Neuropsychiatric Disease and Treatment is an international, peerreviewed journal of clinical therapeutics and pharmacology focusing on concise rapid reporting of clinical or pre-clinical studies on a range of neuropsychiatric and neurological disorders. This journal is indexed on PubMed Central, the 'PsycINFO' database and CAS, and is the official journal of The International Neuropsychiatric Association (INA). The manuscript management system is completely online and includes a very quick and fair peer-review system, which is all easy to use. Visit http://www.dovepress.com/testimonials.php to read real quotes from published authors. 